\section{Guiding you through the rapidly changing world of dentistry}

Henry Schein Dental (HSD), leading global distributor of products and services (including innovative digital dental technology solutions) to office-based dental practitioners, dental specialists and dental laboratories, were on hand at this year's BDIA Dental Showcase to show dentists how they can operate their practices more efficiently.

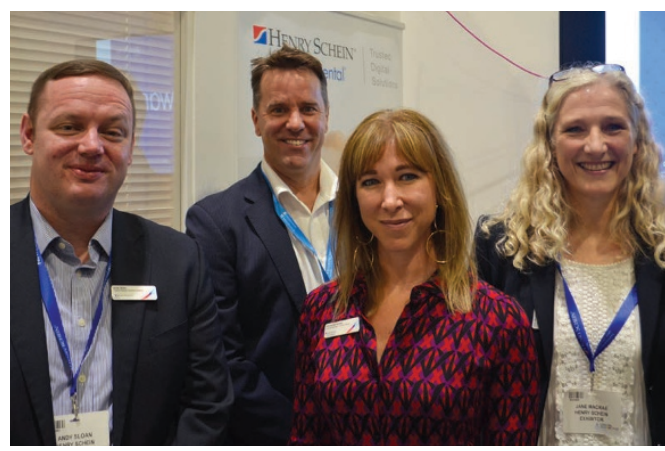

Henry Schein ConnectDental combines workflow optimisation, education, expert advice and support, helping dental professionals navigate their way through the rapidly changing world of digital dentistry.

Henry Schein Dental Laboratory provides the profession with a well-rounded approach encompassing traditional and digital equipment and materials. Specialists are always available to provide service and support, as well as guidance on installations and to answer specific software issues around scanners, milling units or 3D printers.

Henry Schein Business Solutions helps dentists discover more about their individual practice performance by introducing them to Practice Analysis, a programme that is supported by evidence showing that practices that have enrolled in the programme are enjoying an average revenue uplift of $28 \%$, once suggested changes have been implemented.

Henry Schein also rewards customer loyalty and announced at Showcase that since its launch in April this year, Henry Schein Rewards has now enrolled its 1,000th member. The scheme gives members points for every pound spent on almost all Henry Schein Dental and Software of Excellence products and services. It is available to all HSD customers.

For more information visit www.hsdconnectdental.co.uk, www.henryschein.co.uk and www.hsdrewards.co.uk.

\title{
Gain over 3,000 hours of free verifiable CPD
}

In April 2016 GSK, the manufacturer of Corsodyl, Poligrip, Sensodyne and Pronamel, launched four free certified CPD modules. Each provides 1.5 hours of verifiable CPD and so far over 2,000 modules have been completed, meaning GSK has provided over 3,000 hours of free, verifiable CPD to DHCPs across the country.

The modules focus on a range of topics including gum disease and the Basic Periodontal Examination (BPE) with Corsodyl and the impact of an ageing population, the effects of tooth loss and dentures for patients and the role of denture fixatives and cleansers with Poligrip. Sensodyne Repair \& Protect and Pronamel focus on the impacts of dentine hypersensitivity and the mode of action of NovaMin and the impacts of erosive tooth wear and the Basic Erosive Wear Examination (BEWE) respectively.

All modules can be completed remotely at a pace that suits the user. There is a selection of multiple-choice questions

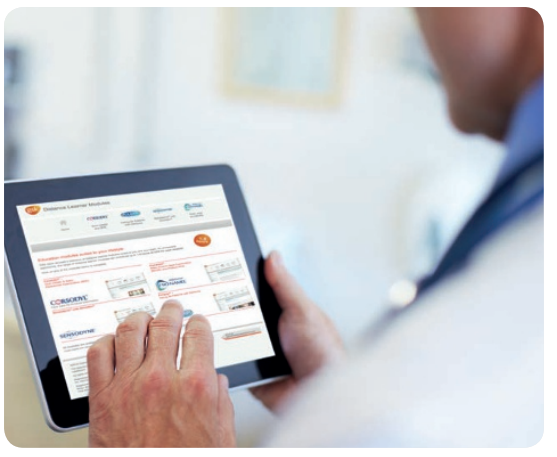

at the end of each module and, upon answering the questions correctly, the user is issued a certificate for completing the CPD module.

GSK sees delivering quality education and CPD as a core part of its mission and strives to continuously meet the needs of DHCPs through online learning as well as face to face lectures.

Access to all modules, as well as information on GSK products, is available at www.gsk-dentalprofessionals.co.uk.

\section{Act now to book your place!}

Places are currently available on some of GC's G-aenial Hands-on and Advanced Courses but they are going fast, so act now to book your place.

As one of the world's most innovative dental companies, GC has continuously made important and ground breaking contributions to dental care. This is coupled with their philosophy to promote communication and understanding between dentists and technicians, with the aim of bringing new concepts, products and methodology to the market.

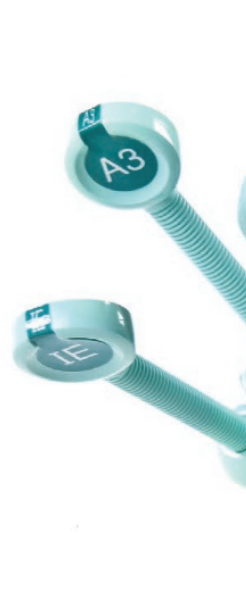

Their commitment to research and continual education has been the foundation for the GC

Europe Campus located on their site in Leuven, Belgium.

This state-of-the-art environment enables GC and their worldwide network of dental professionals to deliver hands-on courses, presentations and demonstrations of all their major technologies and products.

The G-aenial Course programme includes a theoretical presentation on the unique benefits of G-aenial, demonstrations and hands-on sessions for the build-up technique for Class II and IV restorations, theoretical presentations of G-Premio Bond and D-Light Duo, and a demonstration of Gradia Gum. With courses running throughout the year, the course fee includes travel, overnight accommodation, all meals, materials, models and equipment, and ten hours of CPD.

For further information contact GC UK Ltd on 01908 218999, email info@gcukltd. co.uk or visit www.gceurope.com. 